\title{
CANTOR'S INTERSECTION THEOREM AND SOME GENERALIZED FIXED POINT THEOREMS OVER A LOCALLY CONVEX TOPOLOGICAL VECTOR SPACE
}

\author{
A. P. BAISNAB, K. ROY, AND M. SAHA
}

\begin{abstract}
In this present paper, we establish Cantor's intersection like theorem in a locally convex topological vector spaces. Some fixed point and common fixed point theorems are proved for Reich and Caccioppoli type contractive mappings in such a locally convex topological vector space. Also in this setting we prove a fixed point theorem for some mapping which is the uniform limit of a sequence of Reich type contractive mappings therein.

Встановлена теорема, подібна теоремі Кантора про перетин, у випадку локально опуклих векторних просторів. Для стискуючих відображень типу Райха і Каччіополі відповідних просторів доведені теореми про нерухому точку та спільну нерухому точку. Також у цій постановці доведена теорема про нерухому точку для відображення, яке є рівномірною границею послідовності стискуючих відображень типу Райха.
\end{abstract}

\section{INTRODUCTION}

Nowadays fixed point Theory is one of the most emerging areas of research in contemporary Mathematics. The research in this area has been more useful for its numerous applications in various branches in Mathematics, like Solution of nonlinear matrix equations, nonlinear differential and integral equations, boundary value problem, implicit function theorem, homotopy Theory and many more. S. Banach had been first person to prove well known Contraction Principle Theorem [4] in fixed point Theory using contraction mapping in a complete metric space. Since then many researchers had been attracted in fixed point Theory resulting in enormous growth enriching and enlarging frontiers of fixed point theory. By relaxation of spatial structure many researchers had discovered several metric-like structures in underlying spaces and proved important fixed point theorems (See [3], [6], [7], [9], [11] and [16]), where polygonal type inequalities had been extensively used. Attempts have now been in progress to establish fixed point Theorems with Frechet Topological Vector space as underlying space [1]. Very recently Roy and Saha in 2019 (See [13]) have developed fixed point theory in Topological Vector spaces, specially in Locally Convex Topological Vector spaces so far unexplored, and they have proved fixed point theorems involving Kannan type contractive mappings. Since not necessarily continuous mappings are also now welcome in fixed point theory as developed by Roy and Saha [13], we have been prompted to look into problems involving like-Kannan mappings and our investigations in the concerned area have shown that we can derive some interesting and useful too fixed point theorems by taking Reich and Caccioppoli-type mappings over Locally Convex Topological Vector space where CantorIntersection Theorem has been proved. We have not used Minkowski functional over the space in our fixed point theorems. It may now be seen that our findings in fixed point theory choice of a suitable local base in underlying Locally Convex Topological Vector

2020 Mathematics Subject Classification. Primary 47H10; Secondary 54H25.

Keywords. Locally convex topological vector space, Cantor's intersection theorem, fixed point. 
space has played an important part and motivation throughout the text of the paper as presented.

Before we start with actual text we need giving some preliminaries and recall some definitions that help reading the materials with ease and comfort.

\section{Preliminaries}

The following basic definitions and basic results together with its consequences are relevant to our findings.

Definition 2.1. [13] Let $X$ be a real vector space and $C$ be a subset of $X$. Then $C$ is said to be convex if for any two elements $x, y \in C$ and for any scalar $0 \leq \alpha \leq 1$, $\alpha x+(1-\alpha) y \in C$ that is the line segment joining two points $x, y$ must lie in the set $C$. Equivalently, $\alpha C+(1-\alpha) C \subset C$ for all scalars $\alpha$ satisfying $0 \leq \alpha \leq 1$.

Lemma 2.2. [13] $A$ subset $C$ of a vector space $X$ is convex iff for all positive scalars $s$ and $t,(s+t) C=s C+t C$.

Definition 2.3. [13] A subset $S$ of a vector space $X$ is said to be symmetric if $-S \subset S$, equivalently $S=-S$.

Definition 2.4. [13] A subset $B$ of a vector space $X$ is said to be balanced if $\alpha B \subset B$ for all scalars $\alpha$, whenever $|\alpha| \leq 1$.

Definition 2.5. [13] A set $A$ in a vector space $X$ is said to be absorbing if for each $x \in X$ there exists a $\epsilon>0$ such that $\alpha x \in A$, whenever $|\alpha| \leq \epsilon$.

Lemma 2.6. [13] A convex set $C$ of a vector space $X$ is balanced iff it is symmetric.

Definition 2.7. [13] A balanced set $B$ of a vector space $X$ is absorbing iff for each $x \in X$, there corresponds a scalar $\beta \neq 0$ such that $\beta x \in B$.

Definition 2.8. [13] A vector space $X$ over $\mathbb{R}$ or $\mathbb{C}$ equipped with a $T_{1}$ topology $\tau$ is said to be a topological vector space(TVS) if the following conditions are satisfied.

(i) The mapping from $X \times X$ to $X$ defined by $(x, y) \rightarrow x+y, x, y \in X$, is continuous, that is for every neighborhood $W$ of $x+y$ we can find neighborhoods $V_{1}$ of $x$ and $V_{2}$ of $y$ such that $V_{1}+V_{2} \subset W$ and also

(ii) The mapping from $F \times X \rightarrow X$ defined by $(\alpha, x) \rightarrow \alpha x, \alpha \in F, x \in X$, is continuous, that is for any neighborhood $W$ of $\alpha x$ we can find a neighborhood of $\alpha$ say $(\alpha-\delta, \alpha+\delta), \delta>0$ and a neighborhood $V$ of $x$ such that $\gamma V \subset W$ whenever $\gamma \in(\alpha-\delta, \alpha+\delta)$.

We now give the following important and useful Definitions and known results.

Definition 2.9. (Local base)[13] By local base of a TVS $(X, \tau)$ we mean a neighborhood base $\mathbb{B}$ of $\theta \in X(\theta$ is the zero vector in $X$ ) that is for every neighborhood $V$ of $\theta$ there exists a member $B \in \mathbb{B}$ such that $\theta \in B \subset V$.

Definition 2.10. [13] A TVS $X$ is said to be locally convex if $X$ has a local base whose members are all convex sets.

Lemma 2.11. [13] A TVS X has a balanced local base.

Lemma 2.12. [13] Every neighborhood of $\theta$ in a TVS $X$ contains an absorbing neighborhood of $\theta \in X$.

Lemma 2.13. [13] In a locally convex TVS X every neighborhood of $\theta$ contains a absorbing,balanced and convex neighborhood of $\theta$.

Lemma 2.14. [13] Every TVS is regular. 
Lemma 2.15. [13] Let $X$ be a TVS. Then the followings hold.

(i) If $A \subset X$ then $\bar{A}=\cap(A+V)$, where $V \in \mathcal{N}(\theta), \mathcal{N}(\theta)$ is the collection of all neighborhoods of $\theta \in X$.

(ii) If $A \subset X$ and $B \subset X$ then $\bar{A}+\bar{B} \subset \overline{A+B}$.

(iii) If $Y$ is a subspace of $X$ then $\bar{Y}$ is also a subspace of $X$.

(iv) If $C$ is a convex set in $X$ then $\bar{C}$ and $\operatorname{Int}(C)$ are also so.

(v) If $E \subset X$ is balanced then $\bar{E}$ is also balanced, moreover if $\theta \in \operatorname{Int}(E)$ then $\operatorname{Int}(E)$ is also balanced.

(vi) If $A$ is an absorbing subset of $X$ then $\bar{A}$ is also so.

Lemma 2.16. [13] The following conditions are equivalent in a TVS X.

(i) $X$ is $T_{0}$.

(ii) $X$ is $T_{2}$.

(iii) $\cap_{V \in \mathcal{N}(\theta)} V=\{\theta\}$, where $\mathcal{N}(\theta)$ is the collection of all neighborhoods of $\theta \in X$.

Lemma 2.17. [13] In a locally convex TVS X, the balanced, closed, convex neighborhood of $\theta$ forms a neighborhood base of $\theta \in X$.

Definition 2.18. [13] Let $X$ be a TVS. Fix a base $\mathbb{B}$ for $X$. A sequence $\left\{x_{n}\right\}$ in $X$ is said to be a Cauchy sequence if to every $V \in \mathbb{B}$ there corresponds a $N \in \mathbb{N}$ such that $x_{n}-x_{m} \in V$ whenever $m>n \geq N$.

Definition 2.19. [13] A sequence $\left\{x_{n}\right\} \subset X$ is said to be convergent to an element $x \in X$ if for any basic neighborhood $V$, there exists a positive integer $N \in \mathbb{N}$ such that $x_{n}-x \in V$ whenever $m \geq N$ and we write $x_{n} \rightarrow x$ as $n \rightarrow \infty$ and we say that $x$ is the limit of $\left\{x_{n}\right\}$.

Definition 2.20. [13] A TVS $X$ is said to be complete if every Cauchy sequence in $X$ is convergent to an element in $X$.

Lemma 2.21. [13] A TVS X is Hausdorff iff every sequence in $X$ has atmost one limit.

Lemma 2.22. [13] A complete subset of a Hausdorff TVS is closed.

Lemma 2.23. [13] Let $A \subset X$ be complete. Then every closed subset of $A$ is complete.

Definition 2.24. [13] A TVS $X$ is said to be a $F$-space if its topology $\tau$ is induced by a complete invariant metric. A TVS $X$ is a Frechet space if it is a locally convex $F$-space.

Definition 2.25. [13] Let $X$ and $Y$ be two TVSs. Also let $T: X \rightarrow Y$ be a mapping. Then $T$ is said to be continuous at $x_{0} \in X$ if $\left\{x_{n}\right\} \in X$ such that $x_{n} \rightarrow x_{0}$ as $n \rightarrow \infty$ implies $T x_{n} \rightarrow T x_{0}$ as $n \rightarrow \infty$.

In [13] the authors have proved Kannan-type fixed point theorem in the framework of a complete locally convex topological vector space and have applied it to show the stability of fixed point problem.

Definition 2.26. [13] Let $(X, \tau)$ be a locally convex TVS and $T: X \rightarrow X$ be a mapping. Then $T$ is said to be sequentially convergent if for any sequence $\left\{y_{n}\right\}$ in $X$, convergence of $\left\{T y_{n}\right\}$ in $X$ implies that $\left\{y_{n}\right\}$ is convergent in $X$.

Definition 2.27. [13] Let $(X, \tau)$ be a locally convex TVS and $T: X \rightarrow X$ be a mapping. Then $T$ is said to be subsequentially convergent if for any sequence $\left\{y_{n}\right\}$ in $X$, convergence of $\left\{T y_{n}\right\}$ in $X$ implies that $\left\{y_{n}\right\}$ has a convergent subsequence in $X$.

Definition 2.28. [13] Let $(X, \tau)$ be a locally convex TVS and $\left\{T_{n}\right\}$ be a sequence of self maps on $X$. Then $\left\{T_{n}\right\}$ converges uniformly to a self map $T$ on $X$ if for each neighborhood $U$ of $\theta \in X$ there exists $N \in \mathbb{N}$ such that $T_{n} x-T x \in U$ whenever $n \geq N$ for all $x \in X$. 
Lemma 2.29. [13] Let $(X, \tau)$ be a locally convex TVS and $\left\{x_{n}\right\}$ be a sequence in $X$. If for any neighborhood $V$ of $\theta \in X$ there exists some $t>0$ such that for any $n \in \mathbb{N}$, $x_{n}-x_{n+1} \in \alpha^{n} t V$ for some $\alpha \in(0,1)$ then $\left\{x_{n}\right\}$ is Cauchy in $X$.

Definition 2.30. [13] Let $(X, \tau)$ be a locally convex TVS. A mapping $T: X \rightarrow X$ is said to be a Kannan-type contractive mapping if for every neighborhood $U$ of $\theta \in X$ there exists $0<\alpha<\frac{1}{2}$ such that $(T x-T y)-\alpha(y-T y) \in \alpha U$ whenever $x-T x \in U$, $\forall x, y \in X$.

Theorem 2.31. [13] Let $(X, \tau)$ be a complete locally convex topological vector space and let $T: X \rightarrow X$ be a Kannan-type contractive mapping with the constant $\alpha$. Then $T$ has a unique fixed point in $X$.

Theorem 2.32. [13] Let $(X, \tau)$ be a complete locally convex topological vector space and let $T: X \rightarrow X$ be a Kannan-type contractive mapping with the constant $\alpha$. Then the fixed point equation of $T$ is Ulam-Hyers stable.

\section{Cantor's intersection theOREM}

In this section we prove a Cantor's intersection like theorem in the context of locally convex topological vector space.

Theorem 3.1. Let $(X, \tau)$ be a locally convex topological vector space. Then $X$ is complete if and only if the following condition holds:

If $\left\{F_{n}\right\}$ is a decreasing sequence of non-empty closed subsets of $X$ such that for any neighborhood $U$ of $\theta \in X$ there exists some $N \in \mathbb{N}$ such that $F_{N}-F_{N} \subset U$ then $\cap_{n=1}^{\infty} F_{n}$ is a singleton in $X$.

Proof. First let us suppose that $X$ is complete and $\left\{F_{n}\right\}$ is a decreasing sequence of nonempty closed subsets in $X$. Take a neighborhood $V$ of $\theta \in X$. Without loss of generality we can assume that $V$ is convex, balanced and absorbing. Then by the assumed condition there exists some $N \in \mathbb{N}$ such that $F_{N}-F_{N} \subset V$. Therefore $V \supset F_{N}-F_{N} \supset F_{N+1}-F_{N+1} \supset \ldots$. Suppose $m>n \geq N$, then we have $x_{n}-x_{m} \in$ $F_{n}-F_{n} \subset F_{N}-F_{N} \subset V$, which implies $\left\{x_{n}\right\}$ is a Cauchy sequence in $X$ and therefore there exits some $u \in X$ such that $\left\{x_{n}\right\}$ converges to $u$. For any $n \in \mathbb{N}$ we have $x_{m} \in F_{n}$ whenever $m \geq n$. Since $F_{n}$ is closed we get $u \in F_{n}$. Hence $u \in \cap_{n=1}^{\infty} F_{n}$.

Suppose that $w \in \cap_{n=1}^{\infty} F_{n}$. For any neighborhood $U$ of $\theta \in X$ there exists $N_{0} \in \mathbb{N}$ such that $F_{N_{0}}-F_{N_{0}} \subset U$ and therefore $u-w \in F_{N_{0}}-F_{N_{0}} \subset U$. Since $U$ is arbitrary neighborhood of $\theta$ in $X$ we have $u-w=\theta$ implies $u=w$. Therefore $\cap_{n=1}^{\infty} F_{n}=\{u\}$.

Conversely suppose that the condition holds and $\left\{x_{n}\right\}$ is a Cauchy sequence in $X$. Put, $H_{n}=\left\{x_{n}, x_{n+1}, \ldots\right\}$ for all $n \in \mathbb{N}$. Then $\left\{\overline{H_{n}}\right\}$ is a decreasing sequence of nonempty closed sets in $X$. Let $V$ be a neighborhood of $\theta \in X$. Since $\left\{x_{n}\right\}$ is Cauchy there exists $N \in \mathbb{N}$ such that $x_{n}-x_{m} \in \frac{1}{2} V$, whenever $n, m \geq N$. Thus $H_{N}-H_{N} \subset \frac{1}{2} V$. Also let $a, b \in \overline{H_{N}} \backslash H_{N}$ then there exists $\left\{x_{n_{k}}\right\}$ and $\left\{x_{n_{k}}\right\}$ in $H_{N}$ and $x_{n_{k}} \rightarrow a, x_{\dot{n}_{k}} \rightarrow b$ as $k \rightarrow \infty$.

Now,

$$
a-b=\left(a-x_{n_{k}}\right)+\left(x_{n_{k}}-x_{\dot{n}_{k}}\right)+\left(x_{\dot{n}_{k}}-b\right) \text { for all } k \in \mathbb{N} .
$$

By a suitable choice of $k$ we can show that $a-b \in V$. Also if $p \in H_{N}$ and $q \in \overline{H_{N}} \backslash H_{N}$ then there exists some $\left\{x_{m_{k}}\right\} \in H_{N}$ such that $x_{m_{k}} \rightarrow q$ as $k \rightarrow \infty$, and we have

$$
p-q=\left(p-x_{m_{k}}\right)+\left(x_{m_{k}}-q\right) \text { for all } k \in \mathbb{N} .
$$

Similarly as above we can show that $p-q \in V$. From this it follows that $\overline{H_{N}}-\overline{H_{N}} \subset V$. Therefore by the assumed condition we have $\cap_{n=1}^{\infty} \overline{H_{n}}=\{z\}$ for some $z \in X$. Let $W$ be a neighborhood of $\theta \in X$, then there exists $N_{0} \in \mathbb{N}$ such that $\overline{H_{N_{0}}}-\overline{H_{N_{0}}} \subset W$ and 
therefore $x_{n}-z \in W$ whenever $n \geq N_{0}$. So $\left\{x_{n}\right\}$ is convergent in $X$ and converges to $z$. Hence $X$ is complete.

\section{Main RESUlts}

In this section following the literatures [2], [5], [8], [10], [12], [14] and [15] we define Reich-type contractive mapping, Caccioppoli-type contractive mapping, $T$-Reich-type contractive mapping and $T$-Caccioppoli-type contractive mapping over a locally convex TVS and with these mappings we have been able to prove some fixed point theorems and common fixed point theorems over it.

Definition 4.1. Let $(X, \tau)$ be a locally convex TVS. A mapping $T: X \rightarrow X$ is said to be a Reich-type contractive mapping if for every neighborhood $U$ of $\theta \in X$ there exists $\alpha, \beta \geq 0$ with $0<\alpha+2 \beta<1$ such that $(T x-T y)-\beta[(x-T x)+(y-T y)] \in \alpha U$ whenever $x-y \in U, \forall x, y \in X$.

Definition 4.2. Let $(X, \tau)$ be a locally convex TVS and $T: X \rightarrow X$ be a mapping. Then a mapping $S: X \rightarrow X$ is said to be a $T$-Reich-type contractive mapping if there exists $\alpha, \beta \geq 0$ with $0<\alpha+2 \beta<1$ such that $(T S x-T S y)-\beta[(T x-T S x)+(T y-T S y)] \in \alpha U$ whenever $T x-T y \in U$ for all $x, y \in X$ and for any neighborhood $U$ of $\theta \in X$.

Definition 4.3. Let $(X, \tau)$ be a locally convex TVS. A mapping $T: X \rightarrow X$ is said to be a Caccioppoli-type contractive mapping if $T$ satisfies the following conditions:

(i) $a_{n}>0$ for all $n \in \mathbb{N}$ and $\sum_{n=1}^{\infty} a_{n}<\infty$,

(ii) for every neighborhood $U$ of $\theta \in X, T^{m} x-T^{m} y \in a_{m} U$ whenever $x-y \in U$, $\forall x, y \in X$ and for any $m \in \mathbb{N}$.

Definition 4.4. Let $(X, \tau)$ be a locally convex TVS and $T: X \rightarrow X$ be a mapping.. A mapping $S: X \rightarrow X$ is said to be a $T$-Caccioppoli-type contractive mapping if $S$ satisfies the following conditions:

(i) $a_{n}>0$ for all $n \in \mathbb{N}$ and $\sum_{n=1}^{\infty} a_{n}<\infty$,

(ii) for every neighborhood $U$ of $\theta \in X, T S^{m} x-T S^{m} y \in a_{m} U$ whenever $T x-T y \in U$, $\forall x, y \in X$ and for any $m \in \mathbb{N}$.

Theorem 4.5. Let $(X, \tau)$ be a complete locally convex topological vector space and let $T: X \rightarrow X$ be a Reich-type contractive mapping with constants $\alpha, \beta$ (See Definition 4.1). Then $T$ has a unique fixed point in $X$.

Proof. Let $x_{0} \in X$ and let $U$ be a neighborhood of $\theta \in X$. Let us define a sequence $\left\{x_{n}\right\}$ in $X$ by $x_{n}=T x_{n-1}=T^{n} x_{0}$ for all $n \in \mathbb{N}$. We may assume that $U$ is convex, balanced and absorbing. Now $x_{0}-x_{1} \in X$. So there exists a scalar $\lambda>0$ such that $x_{0}-x_{1} \in \eta U$ whenever $|\eta| \geq \lambda$. As $x_{0}-x_{1} \in \lambda U$ then $\left(T x_{0}-T x_{1}\right)-\beta\left[\left(x_{0}-T x_{0}\right)+\left(x_{1}-T x_{1}\right)\right] \in \alpha \lambda U$ that is $x_{1}-x_{2} \in \frac{(\alpha+\beta)}{1-\beta} \lambda U$. Proceeding in a similar fashion we get $x_{n}-x_{n+1} \in\left(\frac{(\alpha+\beta)}{1-\beta}\right)^{n} \lambda U$ for all $n \in \mathbb{N}$. Then by Lemma 2.29 we see that $\left\{x_{n}\right\}$ is Cauchy in $X$. Since $X$ is complete, so there exists $z \in X$ such that $x_{n} \rightarrow z$ as $n \rightarrow \infty$.

Let $V$ be a neighborhood of $\theta \in X$. Then there exists a balanced, convex and absorbing neighborhood $W$ of $\theta \in X$ such that $W \subset \frac{1-\beta}{1+\alpha+\beta} V$. Now since $x_{n} \rightarrow z$ as $n \rightarrow \infty$ so there exists $N \in \mathbb{N}$ such that $x_{n}-z \in W$ and $x_{n}-x_{n+1} \in W$ for all $n \geq N$. So for all $n \geq N$

$$
\begin{aligned}
(z-T z) & -\beta(z-T z) \\
& =z-x_{n+1}+x_{n+1}-T z-\beta\left(x_{n}-T x_{n}\right)+\beta\left(x_{n}-T x_{n}\right)-\beta(z-T z) \\
& =\left(z-x_{n+1}\right)+\left\{\left(T x_{n}-T z\right)-\beta\left[\left(x_{n}-T x_{n}\right)+(z-T z)\right]\right\}+\beta\left(x_{n}-x_{n+1}\right) \\
& \in W+\alpha W+\beta W \subset(1-\beta) V .
\end{aligned}
$$


Then $(1-\beta)(z-T z) \in(1-\beta) V$ whenever $n \geq N$ that is $z-T z \in V$. Since $V$ is any neighborhood of $\theta \in X$ then we have $T z=z$. Clearly the fixed point of $T$ is unique.

Theorem 4.6. Let $(X, \tau)$ be a complete locally convex topological vector space and $f$ be a continuous self map on $X$. Let $g: X \rightarrow X$ be a mapping such that it commutes with $f$ and satisfies $g(X) \subset f(X)$. If for every neighborhood $U$ of $\theta \in X$ there exists constants $\alpha, \beta \geq 0$ with $0<\alpha+2 \beta<1$ such that $(g x-g y)-\beta[(f x-g x)+(f y-g y)] \in \alpha U$ whenever $f x-f y \in U$ for all $x, y \in X$ then $f$ and $g$ have a unique common fixed point in $X$.

Proof. Let $x_{0} \in X$. Then there exists $x_{1} \in X$ such that $f x_{1}=g x_{0}$. Since $x_{1} \in X$ so there exists $x_{2} \in X$ such that $f x_{2}=g x_{1}$. Continuing in this way we get $f x_{n}=g x_{n-1}$ $\forall n \in \mathbb{N}$. Let us take $\left\{y_{n}\right\} \subset X$ defined by $y_{n}=f x_{n}=g x_{n-1}$ for all $n \in \mathbb{N}$.

Let $U$ be a neighborhood of $\theta$ in $X$. Assume that $U$ is convex, absorbing and balanced. So there exists a $t>0$ such that $y_{1}-y_{2}=f x_{1}-f x_{2} \in \zeta U$ whenever $|\zeta| \geq t$. Therefore $\left(g x_{1}-g x_{2}\right)-\beta\left[\left(f x_{1}-g x_{1}\right)+\left(f x_{2}-g x_{2}\right)\right] \in \alpha t U$ that is $y_{2}-y_{3} \in \frac{(\alpha+\beta)}{1-\beta} t U$. Proceeding in this manner we get $y_{n}-y_{n+1} \in\left(\frac{(\alpha+\beta)}{1-\beta}\right)^{n-1} t U$ for all $n \in \mathbb{N}$. So by applying Lemma 2.29 we see that $\left\{y_{n}\right\}$ is Cauchy sequence in $X$. Since $X$ is complete, so there exists $z \in X$ such that $y_{n} \rightarrow z$ as $n \rightarrow \infty$. Since $f$ is continuous we have $f y_{n} \rightarrow f z$ as $n \rightarrow \infty$. Now $f y_{n}=f g x_{n-1}=g f x_{n-1}=g y_{n-1}$ for all $n \geq 2$. Therefore $g y_{n} \rightarrow f z$ as $n \rightarrow \infty$. Now,

$$
\begin{aligned}
g y_{n}- & g z \\
& =g y_{n}-g z-\beta\left[\left(f y_{n}-g y_{n}\right)+(f z-g z)\right]+\beta\left[\left(f y_{n}-g y_{n}\right)+(f z-g z)\right] \\
& =g y_{n}-g z-\beta\left[\left(f y_{n}-g y_{n}\right)+(f z-g z)\right] \\
& \quad+\beta\left[\left(f y_{n}-g y_{n}\right)+\left(f z-g y_{n}\right)+\left(g y_{n}-g z\right)\right] .
\end{aligned}
$$

Which implies that $g y_{n}-g z=\frac{1}{1-\beta}\left\{\left(g y_{n}-g z\right)-\beta\left[\left(f y_{n}-g y_{n}\right)+(f z-g z)\right]+\beta\left(f y_{n}-\right.\right.$ $\left.\left.f y_{n+1}\right)+\beta\left(f z-f y_{n+1}\right)\right\}$. Since $\left\{f y_{n}\right\}$ converges to $f z$, by a routine calculation we can obtain $g y_{n} \rightarrow g z$ as $n \rightarrow \infty$. Thus $f z=g z$. Let $V$ be a neighborhood of $\theta \in X$. So there exists some $\mu>0$ such that $g z-g^{2} z \in \mu V$, which implies $f z-f(g z) \in \mu V$. Hence, $\left(g z-g^{2} z\right)-\beta\left[(f z-g z)+\left(f(g z)-g^{2} z\right)\right] \in \alpha \mu V$ implying that $g z-g^{2} z \in \alpha \mu V$. Continuing in this way we have $g z-g^{2} z \in \alpha^{n} \mu V$ for all $n \geq 1$. Since $0 \leq \alpha<1$ we have $g z-g^{2} z \in V$. Since $V$ is arbitrary we get $g^{2} z=g z$. So $f(g z)=g(f z)=g^{2} z=g z$. Therefore $g z=a$ (say) is a common fixed point of $f$ and $g$ in $X$. Uniqueness of $a$ is apparent.

Theorem 4.7. Let $(X, \tau)$ be a complete locally convex topological vector space. Also let $T, S: X \rightarrow X$ be two mappings satisfying (i) $(T x-S y)-\beta[(x-T x)+(y-S y)] \in \alpha U$ and (ii) $(S x-T y)-\beta[(x-S x)+(y-T y)] \in \alpha U$ whenever $x-y \in U$, for any $x, y \in X$ and for any neighborhood $U$ of $\theta \in X$, where $\alpha, \beta \geq 0$ with $0<\alpha+2 \beta<1$. Then $T, S$ have a unique common fixed point in $X$.

Proof. Let us choose some $x_{0} \in X$. The sequence $\left\{x_{n}\right\}$ in $X$ is defined by

$$
x_{n}= \begin{cases}T x_{n-1}, & \text { when } n \text { is odd } \\ S x_{n-1}, & \text { when } n \text { is even }\end{cases}
$$

Now let $U$ be any neighborhood of $\theta \in X$. We can assume that $U$ is balanced, absorbing and convex. Now $x_{0}-x_{1} \in X$, so there exists some $l>0$ such that $x_{0}-x_{1} \in \beta U$ whenever $|\beta| \geq l$. Thus we get $x_{0}-x_{1} \in l U$ implying that $\left(T x_{0}-S x_{1}\right)-\beta\left[\left(x_{0}-T x_{0}\right)+\right.$ $\left.\left(x_{1}-S x_{1}\right)\right] \in \alpha l U$ (Using condition (i)). That is $x_{1}-x_{2} \in \frac{(\alpha+\beta)}{1-\beta} l U$, which implies that $\left(S x_{1}-T x_{2}\right)-\beta\left[\left(x_{2}-T x_{2}\right)+\left(x_{1}-S x_{1}\right)\right] \in \alpha \frac{(\alpha+\beta)}{1-\beta} l U$ (Using condition (ii)). Thus $x_{2}-x_{3} \in\left(\frac{(\alpha+\beta)}{1-\beta}\right)^{2} l U$, proceeding in a similar way we have $x_{n}-x_{n+1} \in\left(\frac{(\alpha+\beta)}{1-\beta}\right)^{n} l U$ for all $n \in \mathbb{N}$. So by Lemma $2.29\left\{x_{n}\right\}$ is a Cauchy sequence in $X$, since $X$ is complete 
so there exists $z \in X$ to which $\left\{x_{n}\right\}$ converges. So $\left\{T x_{2 n}\right\}_{n \geq 0}$ converges to $z$ and also $\left\{S x_{2 n-1}\right\}_{n \in \mathbb{N}}$ converges to $z$.

Let $V$ be any neighborhood of $\theta$ in $X$. It can be assumed that $V$ is convex, balanced and absorbing. Then there exists $N \in \mathbb{N}$ such that $x_{2 n}-x_{2 n+1} \in \frac{1-\beta}{\alpha+2 \beta} V, S x_{2 n-1}-z \in \frac{1-\beta}{\alpha+2 \beta} V$ and $T x_{2 n}-z \in \frac{1-\beta}{\alpha+2 \beta} V$ whenever $n \geq N$. Therefore using condition (i) we obtain

$$
\begin{aligned}
\left(T x_{2 n}\right. & -S z) \\
& =\left(T x_{2 n}-S z\right)-\beta\left[\left(x_{2 n}-T x_{2 n}\right)+(z-S z)\right]+\beta\left[\left(x_{2 n}-T x_{2 n}\right)+(z-S z)\right] \\
& =\left(T x_{2 n}-S z\right)-\beta\left[\left(x_{2 n}-T x_{2 n}\right)+(z-S z)\right]+\beta\left[\left(x_{2 n}-x_{2 n+1}\right)+\left(z-T x_{2 n}\right)\right. \\
& \left.+\left(T x_{2 n}-S z\right)\right],
\end{aligned}
$$

which in turn implies that $(1-\beta)\left(T x_{2 n}-S z\right)=\left\{\left(T x_{2 n}-S z\right)-\beta\left[\left(x_{2 n}-T x_{2 n}\right)+(z-\right.\right.$ $S z)]\}+\beta\left[\left(x_{2 n}-x_{2 n+1}\right)+\left(z-T x_{2 n}\right)\right]$ and therefore $\left(T x_{2 n}-S z\right) \in V$ for all $n \geq N$. Since $V$ is arbitrary neighborhood of $\theta$ in $X$ we have $T x_{2 n} \rightarrow S z$ as $n \rightarrow \infty$. Since $X$ is Hausdorff then $S z=z$. In a similar fashion using condition (ii) we get $T z=z$. So $z$ is a common fixed point of $T$ and $S$. Uniqueness of $z$ is also clear.

Theorem 4.8. Let $(X, \tau)$ be a complete locally convex topological vector space and $T$ : $X \rightarrow X$ be an one-one, continuous and subsequentially convergent mapping. If $S$ is a $T$-Reich-type contractive mapping then $S$ has a unique fixed point in $X$. Also if $T$ is sequentially convergent then for each $x_{0} \in X$, the sequence of iterates $\left\{S^{n} x_{0}\right\}$ converges to this fixed point.

Proof. Let, $x_{0} \in X$ and let us construct the sequence $\left\{x_{n}\right\}$ in $X$ by $x_{n}=S x_{n-1}=S^{n} x_{0}$ for all $n \in \mathbb{N}$.

Let $U$ be a neighborhood of $\theta \in X$. Without loss of generality we can assume that $U$ is convex, balanced and absorbing. So there exists $h>0$ such that $T x_{0}-T x_{1} \in \gamma U$ whenever $|\gamma| \geq h$. In particular, $T x_{0}-T x_{1} \in h U$, we get $\left(T S x_{0}-T S x_{1}\right)-\beta\left[\left(T x_{0}-\right.\right.$ $\left.\left.T S x_{0}\right)+\left(T x_{1}-T S x_{1}\right)\right] \in \alpha h U$ implying that $T x_{1}-T x_{2} \in \frac{(\alpha+\beta)}{1-\beta} h U$. Proceeding in this way we get, $T x_{n}-T x_{n+1} \in\left(\frac{(\alpha+\beta)}{1-\beta}\right)^{n} h U$ for all $n \in \mathbb{N}$. Then by Lemma 2.29 we see that $\left\{T x_{n}\right\}$ is Cauchy sequence in $X$ and since $X$ is complete so there exists $a \in X$ such that $\lim T x_{n}=a$. Now since $T$ is subsequentially convergent then there exists a subsequence $\left\{x_{n_{k}}\right\}$ of $\left\{x_{n}\right\}$ such that it is convergent and converges to $b \in X$. Since $T$ is continuous, so $\lim T x_{n_{k}}=T b$, implying that $T b=a$. Also,

$$
\begin{aligned}
& T b- T S b \\
&=\left(T b-T S x_{n_{k}}\right)+\left(T S x_{n_{k}}-T S b\right) \\
&=\left(T b-T S x_{n_{k}}\right)+\left(T S x_{n_{k}}-T S b\right)-\beta\left[\left(T x_{n_{k}}-T S x_{n_{k}}\right)+(T b-T S b)\right] \\
&+\quad \beta\left[\left(T x_{n_{k}}-T S x_{n_{k}}\right)+(T b-T S b)\right] \\
&=\left\{\left(T S x_{n_{k}}-T S b\right)-\beta\left[\left(T x_{n_{k}}-T S x_{n_{k}}\right)+(T b-T S b)\right]\right\}+\left(T b-T x_{n_{k}+1}\right) \\
& \quad+\beta\left[\left(T x_{n_{k}}-T x_{n_{k}+1}\right)+(T b-T S b)\right] .
\end{aligned}
$$

This implies that

$$
\begin{aligned}
(1-\beta)(T b- & T S b) \\
=\{ & \left.\left(T S x_{n_{k}}-T S b\right)-\beta\left[\left(T x_{n_{k}}-T S x_{n_{k}}\right)+(T b-T S b)\right]\right\}+\left(T b-T x_{n_{k}+1}\right) \\
& \quad+\beta\left(T x_{n_{k}}-T x_{n_{k}+1}\right) .
\end{aligned}
$$

Now let $V$ be a neighborhood of $\theta \in X$. Since $\left\{T x_{n}\right\}$ is convergent then there exists $N \in \mathbb{N}$ such that $T x_{n_{k}}-T x_{n_{k}+1} \in \frac{1-\beta}{1+\alpha+\beta} V$ and $T x_{n_{k}}-T b \in \frac{1-\beta}{1+\alpha+\beta} V$ whenever $k \geq N$, Therefore $T b-T S b \in V$. Since $V$ is an arbitrary neighborhood of $\theta \in X$ then we get 
$T S b=T b$. Since $T$ is injective then we have $S b=b$ and therefore $b$ is a fixed point of $S$ in $X$. Uniqueness of $b$ is obvious. The last conclusion is now clear.

Theorem 4.9. Let $(X, \tau)$ be a complete locally convex topological vector space and let $T: X \rightarrow X$ be a Caccioppoli-type contractive mapping (See Definition 4.3). Then $T$ has a unique fixed point in $X$.

Proof. Take $x_{0} \in X$ and construct the Picard iterative sequence $\left\{x_{n}\right\}$ as $x_{n}=T x_{n-1}=$ $T^{n} x_{0}$ for all $n \in \mathbb{N}$. Assume that $T x_{0} \neq x_{0}$. Now let $U$ be a neighborhood of $\theta \in X$. Without loss of generality we can assume that $U$ is convex, balanced and absorbing. So there exists $k>0$ such that $x_{0}-x_{1} \in \tau U$ whenever $|\tau| \geq k$. In particular $x_{0}-x_{1} \in k U$, thus we get $x_{n}-x_{n+1}=T^{n} x_{0}-T^{n} x_{1} \in a_{n} k U$ for all $n \in \mathbb{N}$. Therefore, for any $1 \leq n<m$ we get, $x_{n}-x_{m}=\left(x_{n}-x_{n+1}\right)+\left(x_{n+1}-x_{n+2}\right)+\ldots+\left(x_{m-1}-x_{m}\right) \in$ $\left(a_{n}+a_{n+1}+\ldots+a_{m-1}\right) k U=\sum_{i=n}^{m-1} a_{i} k U$. As $\sum_{i=1}^{\infty} a_{i}<\infty$, we have $\sum_{i=n}^{m-1} a_{i} \rightarrow 0$ as $n \rightarrow \infty$. Therefore $x_{n}-x_{m} \in U$ for all $n, m \geq N$, for some $N \in \mathbb{N}$. Since $U$ is arbitrary neighborhood of $\theta \in X$ we see that $\left\{x_{n}\right\}$ is Cauchy in $X$. Thus for completeness of $X$ there exists some $u \in X$ such that $x_{n} \rightarrow u$ as $n \rightarrow \infty$. Now,

$$
\begin{aligned}
u-T u & =u-x_{n+1}+x_{n+1}-T u \\
& =u-x_{n+1}+\left(T x_{n}-T u\right) \text { for all } n \in \mathbb{N} .
\end{aligned}
$$

Now for any neighborhood $V$ of $\theta \in X$ there exists some $N_{0} \geq 1$ such that $x_{n}-u \in \frac{1}{1+a_{1}} V$ for all $n \geq N_{0}$. Therefore $u-T u \in V$. Since $V$ is arbitrary it follows that $T u=u$. Hence $u$ is a fixed point of $T$.

Now let $v$ be any fixed point of $T$ and $W$ be a neighborhood of $\theta \in X$. Then by the absorbing property of $W$ we can find some $t>0$ such that $u-v \in t W$. Therefore $u-v=T^{n} u-T^{n} v \in a_{n} t W$ for all $n \geq 1$. Since the series $\sum_{i=1}^{\infty} a_{i}$ converges we have $a_{n} \rightarrow 0$ as $n \rightarrow \infty$. Then we can find some $N_{1} \in \mathbb{N}$ such that $a_{n} t<1$ for all $n \geq N_{1}$. Hence $u-v \in W$ and we get $u=v$. Therefore the fixed point of $T$ is unique.

Theorem 4.10. Let $(X, \tau)$ be a complete locally convex topological vector space and $T: X \rightarrow X$ be an one-one, continuous and subsequentially convergent mapping. If $S$ is a T-Caccioppoli-type contractive mapping then $S$ has a unique fixed point in $X$. Also if $T$ is sequentially convergent then for each $x_{0} \in X$, the sequence of iterates $\left\{S^{n} x_{0}\right\}$ converges to this fixed point.

Proof. Let us take an element $x_{0} \in X$ and we construct the sequence $\left\{x_{n}\right\}$ in $X$ by $x_{n}=S x_{n-1}=S^{n} x_{0}$ for all $n \in \mathbb{N}$. Let $U$ be a neighborhood of $\theta \in X$. Without loss of generality we can assume that $U$ is convex, balanced and absorbing. So there exists $r>0$ such that $T x_{0}-T x_{1} \in \zeta U$ whenever $|\zeta| \geq r$. Thus $T x_{0}-T x_{1} \in r U$ and therefore we get $T x_{n}-T x_{n+1}=T S^{n} x_{0}-T S^{n} x_{1} \in a_{n} r U$ for all $n \in \mathbb{N}$. Now for any $1 \leq n<m$ we see that $T x_{n}-T x_{m}=\left(T x_{n}-T x_{n+1}\right)+\left(T x_{n+1}-T x_{n+2}\right)+\ldots+\left(T x_{m-1}-T x_{m}\right) \in$ $\left(a_{n}+a_{n+1}+\ldots+a_{m-1}\right) k U=\sum_{i=n}^{m-1} a_{i} k U$. As $\sum_{i=1}^{\infty} a_{i}$ converges, we have $\sum_{i=n}^{m-1} a_{i} \rightarrow 0$ as $n \rightarrow \infty$. Therefore we can find some $N \geq 1$ such that $T x_{n}-T x_{m} \in U$ for all $n, m \geq N$. Since $U$ is arbitrary we see that $\left\{T x_{n}\right\}$ is Cauchy in $X$. Since $X$ is complete, there exists some $z \in X$ such that $T x_{n} \rightarrow z$ as $n \rightarrow \infty$. As $T$ is subsequentially convergent then there exists a subsequence $\left\{x_{n_{k}}\right\}$ of $\left\{x_{n}\right\}$ such that it is convergent and converges to some $z^{\prime} \in X$. Since $T$ is continuous, so $\lim T x_{n_{k}}=T z^{\prime}$, implies that $T z^{\prime}=z$. Here,

$$
\begin{aligned}
T z^{\prime}-T S z^{\prime} & =T z^{\prime}-T x_{n_{k}+1}+T x_{n_{k}+1}-T S z^{\prime} \\
& =\left(T z^{\prime}-T x_{n_{k}+1}\right)+\left(T S x_{n_{k}}-T S z^{\prime}\right) .
\end{aligned}
$$

Now for any neighborhood $V$ of $\theta \in X$ there exists some $N_{0} \geq 1$ such that $T x_{n_{k}}-T z^{\prime} \in$ $\frac{1}{1+a_{1}} V$ for all $n \geq N_{0}$. Therefore $T z^{\prime}-T S z^{\prime} \in V$. Since $V$ is arbitrary it follows that 
$T z^{\prime}=T S z^{\prime}$. Since $T$ is injective then we have $S z^{\prime}=z^{\prime}$ and therefore $z^{\prime}$ is a fixed point of $S$ in $X$.

Now if $\bar{z}$ be any fixed point of $S$ and $W$ be any neighborhood of $\theta \in X$ then we can get some $\mu>0$ such that $\bar{z}-z^{\prime} \in \mu W$, which implies that $\bar{z}-z^{\prime}=T S^{n} \bar{z}-T S^{n} z^{\prime} \in \mu a_{n} W$ for all $n \in \mathbb{N}$. Therefore, for any $n \in \mathbb{N}, T \bar{z}-T z^{\prime} \in a_{n} \mu W$. Since the series $\sum_{i=1}^{\infty} a_{i}$ converges we have $a_{n} \rightarrow 0$ as $n \rightarrow \infty$. Then we can find some $N_{1} \in \mathbb{N}$ such that $a_{n} \mu<1$ for all $n \geq N_{1}$. Hence $T \bar{z}-T z^{\prime} \in W$ and we get $T \bar{z}=T z^{\prime}$, implying that $\bar{z}=z^{\prime}$. Therefore the fixed point of $S$ is unique. Also the final conclusion clearly holds.

Theorem 4.11. Let $(X, \tau)$ be a complete locally convex topological vector space. Let $\left\{T_{n}\right\}$ be a sequence of Reich-type contractive mappings on $X$ with the same constants $\alpha, \beta \geq 0$ satisfying $0<\alpha+2 \beta<1$, which is uniformly convergent to $T$. Then $T$ is also a Reich-type contractive mapping with the same constants $\alpha, \beta \geq 0$ satisfying $0<\alpha+2 \beta<1$ and it has a unique fixed point in $X$.

Proof. Let $V$ be an arbitrary neighborhood of $\theta \in X$. Also let $K$ be a neighborhood of $\theta \in X$ such that $x-y \in K$ for some $x, y \in X$. Now by Lemma 2.17 there exists a closed, balanced and convex neighborhood $G$ of of $\theta \in X$ such that $x-y \in G \subset K$. For the neighborhood $V$ of $\theta \in X$, there exists $N \in \mathbb{N}$ such that for any $a \in X, T_{n} a-T a \in \frac{1}{2} V$ for all $n \geq N$. Now,

$$
\begin{aligned}
(T x-T y)-\beta[(x-T x) & +(y-T y)] \\
= & (1+\beta)\left(T x-T_{n} x\right)+(1-\beta)\left(T_{n} y-T y\right) \\
& +\left\{\left(T_{n} x-T_{n} y\right)-\beta\left[\left(x-T_{n} x\right)+\left(y-T_{n} y\right)\right]\right\} \text { for all } n \in \mathbb{N} .
\end{aligned}
$$

Therefore we have

$$
\begin{aligned}
(T x-T y)-\beta[(x-T x) & +(y-T y)] \\
=(1 & +\beta)\left(T x-T_{N} x\right)+(1-\beta)\left(T_{N} y-T y\right) \\
& +\left\{\left(T_{N} x-T_{N} y\right)-\beta\left[\left(x-T_{N} x\right)+\left(y-T_{N} y\right)\right]\right\} \in \alpha G+V .
\end{aligned}
$$

Since $V$ is arbitrary we get $(T x-T y)-\beta[(x-T x)+(y-T y)] \in \alpha G \subset \alpha K$. Thus $T$ is also a Reich-type contractive mapping with the same constants $\alpha, \beta \geq 0$ satisfying $0<\alpha+2 \beta<1$. Hence by Theorem $4.5 T$ has a unique fixed point in $X$.

Let us consider the sequence of subsets $\left\{K_{m}\right\}_{m \geq 1}$ of $\mathbb{R}^{n}$, where $K_{m}=B[\theta, m], m \in \mathbb{N}$. Let us take the space $C_{c}^{\infty}\left(K_{m}\right)$ of infinitely differentiable functions on $\mathbb{R}^{n}$ with compact support contained in $K_{m}$. Then $C_{c}^{\infty}\left(K_{m}\right)$ is a Frechet space, where the topology $\tau_{m}$ is built by the family of seminorms given by, for each $r \in \mathbb{N},\|f\|_{r}^{(m)}=\sup _{x \in K_{m}}\left|D^{r} f(x)\right|$ for all $f \in C_{c}^{\infty}\left(K_{m}\right)$. Then from the family of topological spaces $\left\{\left(C_{c}^{\infty}\left(K_{m}\right), \tau_{m}\right): m \in \mathbb{N}\right\}$ we have the natural $L F$-space structure on $C_{c}^{\infty}\left(\mathbb{R}^{n}\right)$. We know that $C_{c}^{\infty}\left(\mathbb{R}^{n}\right)$ with this structure is a complete locally convex TVS but not a Frechet space.

Any mapping $T: X \rightarrow X$, where $X=C_{c}^{\infty}\left(\mathbb{R}^{n}\right)$, of the type $T x=\lambda x$ for all $x \in X$, with $\lambda \in[0,1)$ satisfies the contractive condition of Theorem 4.5 with $\alpha=\lambda$ and $\beta=0$ and thus has a unique fixed point $\theta, \theta$ is the null element, in $X$.

Acknowledgment. The second author acknowledges financial support awarded by the Council of Scientific and Industrial Research, New Delhi, India, through research fellowship for carrying out research work leading to the preparation of this manuscript.

\section{REFERENCES}

[1] A. B. Amar, M. A. Cherif and M. Mnif, Fixed-Point Theory on a Frechet Topological Vector Space, International Journal of Mathematics and Mathematical Sciences, Article ID 390720, 9 pages, (2011). 
[2] A. P. Baisnab, P. Das and S. Pal, Fixed points of operators satisfying various contractive conditions in complete partial metric spaces, Fixed Point Theory, 20 (2019), no. 2, 451-468.

[3] I. A. Bakhtin, The contraction mapping principle in quasi-metric spaces, Functional Analysis, Ulyanovsk. Gos. Ped. Inst, Ulyanovsk, 30 (1989).

[4] S. Banach, Sur les operations dans les ensembles abstraits et leur application aux equations untegrales, Fund. Math., 3 (1922), 133-181.

[5] A. Beiranvand, S. Moradi, M. Omid and H. Pazandeh, Two fixed point theorems for special mappings, arXiv:0903.1504v1, (2009).

[6] A. Branciari, A fixed point theorem of Banach-Caccippoli type on a class of generalized metric spaces, Publ. Math. Debrecen, 57 (2000), 31-37.

[7] S. Czerwik, Contraction mappings in b-metric spaces, Acta Math. Inform. Univ. Ostrav., 1 (1993), $5-11$.

[8] D. Dey and M. Saha, Approximate fixed point of Reich operator, Acta Math. Univ. Comenianae, LXXXII (2013), no. 1, 119-123.

[9] R. George, S. Radenović, K. P. Reshma and S. Shukla, Rectangular b-metric space and contraction principles, J. Nonlinear Sci. Appl., 8 (2015), no. 6, 1005-1013.

[10] G. Jungck, Commuting mappings and fixed points, Amer. Math. Monthly, 83 (1976), no. 4, 261-263.

[11] Z. D. Mitrović and S. Radenović, The Banach and Reich contractions in $b_{v}(s)$-metric spaces, J. Fixed Point Theory Appl., 19 (2017), no. 4, 3087-3095.

[12] S. Reich, Some remarks concerning Contraction mappings, Canad. Math. Bull., 14 (1971), 121-124.

[13] K. Roy and M. Saha, Fixed points of mappings over a locally convex topological vector space and Ulam-Hyers stability of fixed point problems, Novi Sad J. Math., 50 (2020), no. 1, 99-112.

[14] B. E. Rhoades, A Comparison of Various Definitions of Contractive Mappings, Trans. Amer. Math. Soc., 226 (1977), 257-290.

[15] M. Saha and R. N. Mukherjee, Fixed point of mappings with contractive iterates in a 2-metric space, Bull. Pure Appl. Math., 1 (2007), no. 1, 79-84.

[16] T. Suzuki, B. Alamri and L. A. Khan, Some notes on fixed point theorems in v-generalized metric space, Bull. Kyushu Inst. Tech. Pure Appl. Math., 62 (2015), 15-23.

PG Section, Department of Mathematics, Lady Brabourne College, P1/2 Suhrawardy Avenue, Kolkata700017, West Bengal, India.

\section{A. P. BAISNAB: baisnababhoypada@yahoo.com}

Department of Mathematics, The University of Burdwan, Purba Bardhaman-713104, West Bengal, India.

K. RoY: kushal.roy93@gmail.com

Department of Mathematics, The University of Burdwan, Purba Bardhaman-713104, West Bengal, India.

M. SAHA: mantusaha.bu@gmail.com

Received 14/06/2020; Revised 02/07/2020 